\title{
FATORES ASSOCIADOS ÀS QUEDAS ENTRE IDOSOS PRATICANTES DE ATIVIDADES FÍSICAS
}

\author{
Amanda Pacheco Beckㄹ, Danielle Ledur Antes², Simone Teresinha Meurer³, Tânia Rosane Bertoldo Benedetti4, \\ Marize Amorim Lopes ${ }^{5}$
}

\footnotetext{
${ }^{1}$ Graduada em Educação Física. Santa Catarina, Brasil. amandinhabeck@yahoo.com.br

2 Mestranda do Programa de Pós-graduação em Educação Física da Universidade Federal de Santa Catarina (UFSC). Santa Catarina, Brasil. daniantes@yahoo.com.br

${ }^{3}$ Mestre em Educação Física. Santa Catarina, Brasil. simonemeurer@yahoo.com.br

${ }^{4}$ Doutora em Enfermagem. Professora do Centro de Desportos (CDS) da UFSC. Santa Catarina, Brasil. benedetti@cds.ufsc.br

${ }^{5}$ Mestre em Educação Física. Professora do CDS/UFSC. Santa Catarina, Brasil. marizefloripa@hotmail.com
}

\begin{abstract}
RESUMO: Objetivou analisar a incidência e os fatores associados às quedas em idosos praticantes de atividades físicas. A amostra constituiu-se de 28 idosos inseridos no Programa de Atividades Físicas para Terceira Idade do Centro de Desportos da Universidade Federal de Santa Catarina. Trata-se de um estudo descritivo. Para a coleta dos dados utilizou-se entrevista semi-estruturada e estatística descritiva para a análise dos dados. Identificou-se uma baixa porcentagem (7,63\%) de quedas entre os idosos. Os fatores associados às quedas foram: ser do sexo feminino, ter entre 70 e 79 anos e apresentar problemas de visão. A maioria das quedas ocorreu dentro de casa e, após a queda, os idosos tiveram restrições nas suas atividades diárias e, a maioria, relatou medo de sofrer nova queda. O estudo indica que a prática de atividades físicas é um fator de proteção para quedas.
\end{abstract}

DESCRITORES: Acidentes por quedas. Envelhecimento. Atividade física.

\section{FACTORS ASSOCIATED WITH FALLS AMONG ELDERLY PRACTITIONERS OF PHYSICAL ACTIVITIES}

\begin{abstract}
The objective of this descriptive study was to analyze the incidence of and factors associated with falls among elderly who practice physical activities. The sample consisted of 25 elderly who participated in the Elderly Physical Activities Program at the Sports Center of the Federal University of Santa Catarina, Brazil. Data was collected through application of a semi-structured interview and analyzed according to descriptive statistics. A low fall percentage $(7.63 \%)$ was identified among the elderly participants of this study. The factors associated with falling were: female gender, being between 70 to 79 years old, and having vision problems. Most of the falls occurred at home and following their fall, these elderly began to have restrictions in their daily activities. The majority of the sample reported a fear of falling again. The study indicates that physical activity is a protective factor concerning falls.
\end{abstract}

DESCRIPTORS: Accidental falls. Aging. Physical activity.

\section{FACTORES ASOCIADOS CON CAÍDAS ENTRE LOS ANCIANOS PRACTICANTES DE ACTIVIDADES FÍSICAS}

RESUMEN: Este estudio tiene como objetivo analizar la incidencia y los factores asociados con caídas en los ancianos practicantes de actividades físicas. La muestra consistió de 28 ancianos participantes en el Programa de Actividades Físicas para la Tercera Edad, del Centro de Deportes de la Universidad Federal de Santa Catarina. Se trata de un estudio descriptivo. Para la recolección de los datos se utilizó una entrevista semi-estructurada y para el análisis, la estadística descriptiva. Fue detectado un bajo porcentaje $(7,63 \%)$ de caídas en los ancianos. Los factores asociados con las caídas son: el hecho de ser mujer, tener entre 70 y 79 y presentar problemas de visión. La mayoría de las caídas ocurrieron en el hogar. Se detecto también que después de una caida, los ancianos tuvieron restricciones en sus actividades diarias y temores de sufrir otra caída. El estudio indica que la práctica de actividad física es un factor de protección de caídas.

DESCRIPTORES: Accidentes por caídas. Envejecimiento. Actividad física. 


\section{INTRODUÇÃO}

De acordo com a Pesquisa Nacional de Amostra e Domicílio (PNAD), realizada no Brasil em $2007,10,5 \%$ da população brasileira tem idade igual ou superior a sessenta anos. ${ }^{1} \mathrm{O}$ envelhecimento populacional e o aumento na expectativa de vida têm consequências econômicas e sociais consideráveis ${ }^{2}$, trazendo à tona a discussão sobre eventos incapacitantes para essa faixa etária.

A ocorrência de quedas entre os idosos é um dos principais problemas clínicos e de saúde pública que contribuem para a incapacidade das pessoas de faixa etária avançada. As quedas têm alta incidência e geram complicações para a saúde e elevados custos assistenciais. ${ }^{3}$ Queda é definida como um evento não intencional que tem como resultado a mudança de posição do indivíduo para um nível mais baixo em relação à sua postura inicial. ${ }^{4}$ De acordo com a Organização Mundial da Saúde ${ }^{5}$, a queda é um dos problemas mais importantes e comuns relatados entre os idosos e aumenta progressivamente com o avanço da idade em ambos os sexos.

No Brasil, a ocorrência de quedas anualmente atinge $32 \%$ dos idosos entre 65 e 74 anos, $35 \%$ de 75 a 84 anos e $51 \%$ acima de 85 anos. De modo geral, $30 \%$ dos idosos brasileiros caem ao menos uma vez ao $\mathrm{ano}^{6}$, podendo ser fatal nesta faixa etária, pois as mudanças fisiológicas normais associadas à idade dificultam a recuperação. ${ }^{7}$

Mudanças naturais do processo de envelhecimento podem causar limitações funcionais tornando o idoso mais vulnerável à queda. A marcha e a estabilidade postural dependem do bom funcionamento dos sistemas neuromuscular, sensorial, musculoesquelético e sistema nervoso central. ${ }^{8}$ Dentro deste conceito, o "cair" pode ser a manifestação de problemas nestes sistemas ou estar relacionado a fatores extrínsecos. ${ }^{9}$

Além disso, uma queda pode significar um ponto de deterioração global no estado de saúde do idoso. $\mathrm{O}$ risco de morte, no ano seguinte à hospitalização por queda, varia entre $15 \%$ e $50 \% .^{10}$ Entre as pessoas com mais de 75 anos, $7 \%$ são atendidas em pronto-socorro para tratamento de lesões causadas por queda, dessas mais de $40 \%$ necessitam de internação hospitalar, e $40 \%$ dos idosos internados continuam com queixas de dores ou limitação em atividades cotidianas. ${ }^{8}$

Existe uma grande preocupação com a independência e autonomia do idoso após uma queda, pois o impacto causado na realização das atividades cotidianas pode trazer maior dependência, principalmente para realização das atividades intermediárias da vida diária (AIVD). A incapacidade para realização das AIVDs, advindas de imobilidades provocadas pela queda, pode trazer, a longo prazo, não apenas consequências aos idosos, mas também aos serviços de saúde e a seus familiares que precisam se mobilizar para o tratamento e a recuperação do idoso. ${ }^{11}$ Está evidente o aumento na incidência de quedas entre os idosos e os prejuízos que uma queda pode acarretar. Porém, apesar dos vários indicativos apontados pela literatura, ainda existem dúvidas com relação aos fatores associados às quedas em idosos ativos. Diante do exposto, o objetivo deste trabalho foi analisar a incidência e os fatores associados à queda em idosos praticantes de atividades físicas.

\section{MÉTODO}

Esta pesquisa foi aprovada pelo Comitê de Ética para Seres Humanos da Universidade Federal de Santa Catarina, processo $n^{\circ} .050 / 05$. Todos idosos foram avaliados após assinarem o Termo de Consentimento Livre e Esclarecido.

A população do estudo foi composta por 377 idosos participantes do Programa de Atividade Física e Dança Folclórica para a Terceira Idade do Centro de Desportos (CDS) da Universidade Federal de Santa Catarina (UFSC).

O referido programa foi criado no ano de 1985 e tem como objetivo oportunizar a prática de atividades físicas e recreativas, buscando a manutenção da saúde e favorecendo a mudança do estilo de vida. Assim, são oferecidas diferentes modalidades de atividades físicas, compreendendo exercícios sensório-motores, trabalhos aeróbios, de alongamento, flexibilidade, equilíbrio, força, resistência, relaxamento, ritmo, entre outros. O programa atende pessoas acima de 55 anos de ambos os sexos, com frequência semanal de duas a três vezes e com duração de 50 a 60 minutos.

Foi realizado um levantamento, nas fichas cadastrais dos idosos, que são atualizadas anualmente com informações pessoais e também relato de quedas. Para a entrevista foram selecionados todos os indivíduos que sofreram queda no período de janeiro/2008 a maio/2009, totalizando 28 idosos. Todos esses responderam uma entrevista semiestruturada acerca da sua última queda e de informação sobre casos anteriores. A entrevista foi realizada sempre pela mesma pesquisadora, 
de forma individual e no local onde os idosos praticavam atividades físicas.

Os dados foram analisados por meio de estatística descritiva com as medidas de tendência central, dispersão, frequência e tabelas de contingência.

\section{RESULTADOS}

Dentre os idosos que participaram do programa de Atividade Física e Dança Folclórica para a Terceira Idade do CDS, no período de janeiro/2008 a maio/2009, foi identificada uma prevalência de $7,63 \%$ de quedas.

Na tabela 1, pode ser visualizada a média de idade dos dois grupos (com e sem relato de quedas), frequência de homens e mulheres e distribuição dos idosos de acordo com as faixas etárias.

Observa-se, na tabela 1 que a média de idade dos idosos que relataram quedas foi mais alta. As idosas que caíram estavam principalmente na faixa etária de 70 a 79 anos, enquanto as que não caíram pertenciam à faixa etária de 60 a 69 anos (idosos mais jovens). Somente as mulheres relataram queda, porém vale ressaltar que o número de homens participantes do programa de atividade física do CDS/UFSC é reduzido.

Tabela 1 - Média de idade, frequência por faixa etária e sexo dos idosos que não caíram (não queda) e dos que caíram (queda), participantes do Programa de Atividades Físicas para Terceira Idade do Centro de Desportos da Universidade Federal de Santa Catarina. Florianópolis-SC, 2009

\begin{tabular}{|c|c|c|c|c|}
\hline \multirow{3}{*}{ Média de idade } & \multirow{2}{*}{\multicolumn{2}{|c|}{$\begin{array}{c}\text { Não queda } \\
69,9 \\
(\mathrm{DP}=5,91)\end{array}$}} & \multirow{2}{*}{\multicolumn{2}{|c|}{$\begin{array}{c}\text { Queda } \\
72,14 \\
(\mathrm{DP}=5,98)\end{array}$}} \\
\hline & & & & \\
\hline & $\mathbf{n}$ & $\%$ & $n$ & $\%$ \\
\hline $60-69$ anos & 171 & 50,4 & 8 & 28,6 \\
\hline $70-79$ anos & 144 & 42,5 & 18 & 64,3 \\
\hline 80 anos ou mais & 24 & 7,1 & 2 & 7,1 \\
\hline Mulheres & 293 & 86,4 & 28 & 100 \\
\hline Homens & 46 & 13,6 & - & - \\
\hline
\end{tabular}

Outro fator importante a ser considerado é a presença de quedas anteriores. Das 28 idosas que relataram queda, a maior parte $(\mathrm{n}=19)$ apresentou também queda anterior ao período investigado. O que mais se destaca é o fato de que metade dos idosos ( $n=14)$ caíram apenas uma vez e sete idosas caíram duas vezes. Ressalta-se que duas idosas relatam mais de dez quedas no período de um ano.
Com relação às causas ou fatores de risco para quedas em idosos observa-se com frequência os problemas de visão, utilização de óculos para todas as atividades diárias e a percepção subjetiva de visão dos idosos com relato de quedas. Apenas um entrevistado não relatou problemas de visão. A maioria $(n=17)$ utiliza óculos para a realização das atividades cotidianas. Com relação à percepção subjetiva da visão, 17 idosos se classificaram como regulares, sete como boas ou muito boas e quatro como ruins ou muito ruins.

Na tabela 2, estão identificados os locais em que ocorreram as quedas, sendo que a maior incidência aconteceu em casa (dentro ou nas dependências da casa), seguida por quedas na rua longe de casa. Em geral, os idosos tropeçaram em calçadas irregulares ou escorregaram em piso molhado. Destaca-se que a maior parte das quedas aconteceu quando os idosos caminhavam, como forma de deslocamento.

Tabela 2 - Local, circunstância, razão da queda e atividade no momento da queda dos idosos participantes do Programa de Atividades Físicas para Terceira Idade do Centro de Desportos da Universidade Federal de Santa Catarina. Florianópolis-SC, 2009

\begin{tabular}{lr}
\hline Variáveis & $\mathbf{n}$ \\
\hline Local da queda & \\
Casa & 13 \\
Rua - próximo de casa & 6 \\
Rua - longe de casa & 9 \\
Circunstância da queda & \\
Tropeçou & 15 \\
Escorregou & 9 \\
Outro (empurrada, virou o pé, pisou em falso) & 3 \\
Não lembra & 1 \\
Razão da queda & \\
Calçada irregular & 11 \\
Local molhado & 5 \\
Degrau ou rampa & 4 \\
Tapete & 1 \\
Não lembra & 7 \\
Atividade no momento da queda & \\
Caminhando (deslocamento) & 16 \\
Correndo para pegar ônibus & 2 \\
Trabalho doméstico & 6 \\
Escada (subindo ou descendo) & 3 \\
Alongamento & 1 \\
\hline
\end{tabular}

Além da investigação dos fatores associados, buscou-se identificar as consequências das quedas. 
A tabela 3 apresenta as principais consequências, desde o momento de atendimento no local da queda ou ajuda para levantar, até as lesões decorrentes e os problemas advindos da queda para a realização das atividades da vida diária

Verifica-se na tabela 3 que nenhum idoso precisou de atendimento médico no local da queda, e praticamente a metade necessitou de ajuda para levantar do chão. Identificou-se que metade dos idosos que caíram realizaram consulta médica para tratamento ou investigação sobre as consequências e lesões da queda, e a outra metade relatou não necessitar de atendimento médico após a queda.

Tabela 3 - Frequência das idosas participantes do Programa de Atividade Física do Centro de Desportos da Universidade Federal de Santa Catarina, que necessitaram de ajuda e atendimento médico, mudanças nas AVDs e medo de cair, entre outras consequências. Florianópolis-SC, 2009

\begin{tabular}{|c|c|c|}
\hline \multirow[t]{2}{*}{ Variáveis } & \multicolumn{2}{|c|}{$\begin{array}{c}\text { Frequência } \\
\text { absoluta }\end{array}$} \\
\hline & Sim & Não \\
\hline \multicolumn{3}{|l|}{ Ajuda e atendimento médico } \\
\hline Atendimento médico local & - & 28 \\
\hline Ajuda para levantar & 12 & 16 \\
\hline Atendimento médico após a queda & 14 & 14 \\
\hline \multicolumn{3}{|l|}{$\begin{array}{l}\text { Mudança nas AVDs após a queda } \\
\text { e medo de cair novamente }\end{array}$} \\
\hline $\begin{array}{l}\text { Mudança após a queda } \\
\text { (restrição nas AVDs) }\end{array}$ & 12 & 16 \\
\hline Medo de cair novamente & 22 & 6 \\
\hline $\begin{array}{l}\text { Mudanças nas AVDs por medo de } \\
\text { nova queda }\end{array}$ & 2 & 26 \\
\hline $\begin{array}{l}\text { Consequências } \\
\text { (lesões) }\end{array}$ & \multicolumn{2}{|c|}{$\begin{array}{l}\text { Frequência } \\
\text { absoluta }\end{array}$} \\
\hline Nenhum machucado & \multicolumn{2}{|c|}{3} \\
\hline Escoriações e hematomas & \multicolumn{2}{|c|}{12} \\
\hline Escoriações e torção & \multicolumn{2}{|c|}{6} \\
\hline Corte e escoriações & \multicolumn{2}{|c|}{2} \\
\hline Torção & \multicolumn{2}{|c|}{2} \\
\hline Fratura & \multicolumn{2}{|c|}{1} \\
\hline Fratura e cirurgia & \multicolumn{2}{|c|}{1} \\
\hline Corte, escoriações e fratura & \multicolumn{2}{|c|}{1} \\
\hline
\end{tabular}

Com relação às mudanças consequentes da queda, 16 idosos mencionaram ter restringido, ao menos temporariamente, as suas atividades diárias. A maioria dos idosos relatou medo de sofrer nova queda, mas apenas dois deixaram de fazer algumas atividades em decorrência do medo.

\section{DISCUSSÃO}

O presente estudo identificou incidência de $7,63 \%$ de quedas em idosos praticantes de atividades físicas. Outro trabalho que investigou a ocorrência de quedas em idosos praticantes de atividades físicas encontrou prevalência superior ao da presente investigação, verificando ocorrência de quedas em $11,4 \%$ dos idosos. ${ }^{12}$

Estudos que não consideraram a prática de atividade física também encontraram percentuais mais elevados de ocorrência de quedas, quando comparados a presente investigação, tanto em âmbito nacional quanto internacional. ${ }^{3,13-14} \AA$ exemplo, um estudo que identificou frequência de quedas em 34,8\% dos idosos residentes nas áreas de abrangência de unidades básicas de saúde de 41 municípios brasileiros com mais de 100 mil habitantes. ${ }^{13}$ Houve ainda uma investigação, realizada com idosos residentes em São Paulo, que encontrou prevalência de $31 \%$ de quedas no período de um ano. ${ }^{3}$

Um trabalho internacional ${ }^{14}$ analisou a prevalência de quedas entre as sete cidades dos estudos SABE (Salud, Bienestar y Envejecimiento en América Latina y el Caribe) e H-EPESE (Hispanic Established Populations for Epidemiologic Studies of the Elderly). A ocorrência de quedas ficou entre 21,6\% em Bridgetown, Barbados, e 34,0\%, em Santiago, Chile. Em estudo ${ }^{14}$ realizado com 1100 idosas britânicas, 27,7\% narraram ter sofrido queda nos 12 meses que antecederam a pesquisa.

$\mathrm{Na}$ maioria dos estudos realizados com idosos não praticantes de atividades físicas, foram encontrados percentuais mais elevados de incidência de quedas. Assim, pode-se sugerir que a atividade física influencia de forma preventiva na ocorrência destes acidentes.

Com relação ao sexo, verificou-se que somente as mulheres foram acometidas por quedas, essa afirmação pode estar vinculada ao prevalente número de mulheres $(86,4 \%)$ participantes do programa investigado, enquanto os homens representam 15,6\%. Esses resultados corroboram com vários outros estudos que indicam prevalência de $60 \%$ a $100 \%$ de queda em mulheres. ${ }^{11,13-14,16-17}$ Em um trabalho de revisão que abordou quinze estudos também foi encontrado que ser do sexo feminino é um dos principais fatores associados ao aumento de risco para quedas. ${ }^{18}$

No presente estudo, a maior frequência de quedas foi relatada pelos idosos que se enquadram na faixa etária de 70 a 79 anos, corroborando com 
outros trabalhos que identificaram maior índice de quedas na mesma faixa etária. ${ }^{12,16-17}$ A ocorrência de quedas tende a aumentar linearmente com a idade, ${ }^{6,18}$ sugerindo que os idosos jovens conseguem desempenhar suas atividades sem grandes alterações em função do processo de envelhecimento. Já, a partir dos 70 anos, acontecem perdas mais acentuadas nas capacidades físicas e as limitações aumentam.

O aumento da ocorrência de quedas, conforme avança a idade cronológica, acontece devido aos efeitos cumulativos das alterações relacionadas à idade, às doenças e ao meio-ambiente inadequado. ${ }^{6}$ Com o envelhecimento ocorre perda de células musculares e elasticidade dos tecidos, diminuição da massa óssea, alterações posturais e redução da mobilidade articular ${ }^{7}$, sistemas esses responsáveis pela estabilidade do corpo, portanto a degradação desses componentes parece predispor o idoso a uma maior incidência de queda.

As entrevistas apontaram que 14 idosos $(50 \%)$ relataram duas ou mais quedas. Esses dados são superiores aos mencionados, em estudo de coorte com 1.667 idosos, no qual foi observado $11 \%$ de relatos de duas ou mais quedas. ${ }^{3}$ Os mesmos autores mencionaram o comprometimento da visão como sendo um fator de risco para a ocorrência destes acidentes, fato igualmente identificado nesta investigação, na qual 27 idosos que caíram narraram ter algum problema de visão.

Outros trabalhos também mencionam que a diminuição da visão (redução da percepção de distância e visão periférica e adaptação ao escuro) é considerada fator intrínseco para a ocorrência de quedas. ${ }^{6,9-10,19}$

Em relação aos fatores extrínsecos para quedas, que são os comportamentos e atividades das pessoas idosas no meio ambiente, ${ }^{20}$ as causas de queda mais mencionadas na presente investigação foram as irregularidades na calçada $(n=11)$, seguida pelo fato do local estar molhado $(n=5)$. As atividades desenvolvidas no momento da queda, citadas com maior frequência, foram o deslocamento $(n=16)$ e a realização de algum trabalho doméstico $(n=6)$. Os locais onde ocorreram o maior número de quedas foi dentro de casa $(n=9)$ e em ruas distantes da residência $(n=7)$.

Estes resultados corroboram com a Organizações de Saúde Internacionais, ${ }^{21}$ que mencionam que a maioria das quedas são acidentais e ocorrem dentro de casa ou em seus arredores, geralmente, durante o desempenho de atividades cotidianas como caminhar, mudar de posição e ir ao ba- nheiro. $\mathrm{O}$ fato dos idosos diminuírem a atenção durante a realização das tarefas, ou estarem com pressa em desenvolver as atividades, os deixa mais vulneráveis a acidentes. Os fatores relacionados ao ambiente físico são responsáveis por 30 a $50 \%$ das quedas e são as causas mais comuns entre idosos. ${ }^{6,19-21}$

Investigação sobre os fatores que ocasionaram quedas em 2.193 pessoas, com idades a partir de 45 anos, revelou que a maioria das quedas (73\%) foi ocasionada por fatores ambientais, tais como superfícies irregulares e tropeçar ou escorregar em objetos, e andar a pé $(47,3 \%)$ foi o fator mais citado. ${ }^{22}$

A partir dos resultados encontrados em relação às causas das quedas, considera-se que muitas poderiam ser prevenidas por meio de mudança de pequenos hábitos, como evitar tapetes soltos pela casa, prestar maior atenção ao caminhar em ruas/calçadas irregulares e durante a realização de atividades domésticas, utilizar o corrimão para subir e descer escadas e usar calçados e roupas adequadas.

Percebeu-se que, entre os idosos que caíram, houve alta prevalência de medo de cair novamente (78,57\%), bem como mudanças de hábitos de alguma das AVDs (45,85\%). Esses resultados condizem com os identificados em um estudo realizado com setenta e dois idosos, o qual identificou que $88,5 \%$ tem medo de cair novamente, $26,9 \%$ relataram abandono de atividades e $23,1 \%$ modificaram seus hábitos em virtude da queda. ${ }^{23}$

Quanto às consequências das quedas, o presente estudo identificou como principais: as escoriações, hematomas e torções. Esses resultados diferem dos identificados por outro estudo que mostrou as fraturas como consequência mais comum. ${ }^{23}$ Desta forma, verifica-se que os idosos participantes de atividades físicas tiveram consequências mais leves, o que pode estar relacionado ao estilo de vida ativo.

Cerca de $4 \%$ das quedas resultam em fraturas e $11 \%$ ocasionaram outras lesões graves, como ferimentos na cabeça, lesões dos tecidos moles e graves ferimentos. ${ }^{24}$ Devido a essas lesões os idosos que sobrevivem à queda comumente restringem suas atividades. Além das consequências físicas ocasionadas pelas lesões, consequências psicológicas também devem ser consideradas. $\mathrm{O}$ medo de cair ainda conduz muitos idosos a diminuírem suas atividades. Tais razões podem acarretar menor confiança na capacidade de caminhar levando o idoso a um maior declínio 
funcional, depressão, sentimentos de desamparo, isolamento social e, consequentemente, a uma perda da qualidade de vida. ${ }^{24}$

\section{CONSIDERAÇÕES FINAIS}

O presente estudo, quando comparado com outras investigações, identificou baixa prevalência de quedas $(7,63 \%)$ entre idosos, o que permite sugerir a prática de atividades físicas como um possível fator de proteção para quedas.

Os fatores associados às quedas foram: ser do sexo feminino, ter entre 70 e 79 anos, apresentar problemas de visão e também fatores ambientais como locais molhados (dentro de casa) e calçadas irregulares (quando caíram na rua).

Diante desses resultados, torna-se visível a importância dos programas de atividade física para estimular a melhoria das capacidades funcionais dos idosos, utilizando-se, além dos exercícios físicos propriamente ditos, de exercícios que simulem as atividades da vida diária, o que minimizaria o risco de quedas.

Visto os altos custos que uma queda causa aos cofres públicos, bem como as implicações sobre a qualidade de vida dos envolvidos, há necessidade das políticas públicas atentarem-se para a redução das barreiras arquitetônicas, especialmente as calçadas irregulares, com o intuito de diminuir a ocorrência de quedas e, consequentemente, os gastos. Além disso, evidencia-se a necessidade de políticas para ampliar e melhorar os programas de atividades físicas destinados à faixa etária em questão.

\section{REFERÊNCIAS}

1. Instituto Brasileiro de Geografia e Estatística. Pesquisa nacional por amostra de domicílios Síntese de indicadores, 2007 [pagina na Internet]. Rio de Janeiro (RJ): IBGE; 2008 [acesso 2009 Abr 2009]. Disponível em: www.ibge.gov.br/home/ estatistica/populacao/trabalhoerendimento/ pnad2007/sintesepnad2007.pdf

2. Benedetti TRB, Gonçalves LHT, Mota JAPS. Uma proposta de política pública de atividade física para idosos. Texto Contexto Enferm. 2007 Jul-Set; 16(3):387-98

3. Perracini M, Ramos L. Fatores associados a quedas em uma coorte de idosos residentes na comunidade. Rev Saúde Pública. 2002 Dez; 36(6):709-16.

4. Moura RN, Santos FCD, Driemeier M, Santos LMD, Ramos LR. Quedas em idosos: fatores de risco associados. Gerontologia. 1999; 7(2):15-21.
5. Organização Mundial da Saúde. Envelhecimento ativo: um projeto de política de saúde. Espanha: OMS; 2002.

6. Pereira SEM, Buksman S, Perracini M, Py L, Barreto KML, Leite VMM. Quedas em idosos. Sociedade Brasileira de Geriatria e Gerontologia. Associação Médica Brasileira e Conselho Federal de Medicina - Projeto Diretrizes; 2001.

7. Hayflick L. Como e por que envelhecemos. Rio de Janeiro: Campus; 1997.

8. Britto FC, Costa SMN. Quedas. In: Papaleo Netto M, Brito FC. Urgências em geriatria. São Paulo: Ed. Atheneu; 2001. p. 323-35

9. Barbosa M. Como avaliar queda em idosos? Rev Assoc Med Bras. 2001 Abr-Jun; 47(2): 93-4.

10. Baraff L, Della Penna R, Williams N, Sanders A. Practice guideline for the ED management of falls in community-dwelling elderly persons. Ann Emerg Med. 1997 Oct; 30(4):480-92.

11. Fabrício SCC, Rodrigues RAP, Costa JML. Causas e conseqüências de quedas de idosos atendidos em hospital público. Rev Saúde Pública. 2004 Feb; 38(1):93-9.

12. Benedetti TRB, Binotto MA, Petroski EL, Gonçalves LHT. Atividade física e prevalência de quedas em idosos residentes no sul do Brasil. Rev Bras de Geriatr Gerontol. 2008 Mai; 11(2):145-54.

13. Siqueira F, Facchini L, Piccini R, Tomasi E, Thumé E, Silveira D, et al. Prevalência de quedas em idosos e fatores associados. Rev Saúde Pública, 2007 Out; 41(5):749-56.

14. Reyes-Ortiz C, Al Snih S, Markides K. Falls among elderly persons in Latin America and the Caribbean and among elderly Mexican-Americans. Rev Panam Salud Publica. 2005 Mai; 17(5-6):362-9.

15. Iglesias C, Manca A, Torgerson D. The health-related quality of life and cost implications of falls in elderly women. Osteoporos Int. 2009 Jun; 20(6):869-78.

16. Fabrício SCC, Rodrigues RAP. Percepção de idosos sobre alterações das atividades da vida diária após acidentes por queda. R Enferm UERJ. 2006 Dez; 14(4):531-7.

17. Hamra A, Ribeiro MB, Miguel OF. Comparação entre fratura por queda em idosos e uso prévio de medicamentos. Acta Ortop Bras. 2007; 15(3):143-5.

18. Gama Z, Gómez-Conesa A. Factores de riesgo de caídas en ancianos: revisión sistemática. Rev Saúde Pública. 2008 Out; 42(5):946-56.

19. Jahana KO, Diogo MJD. Quedas em idosos: principais causas e conseqüências. Saúde Coletiva. 2007; 4(17):148-53.

20. Ministério da Saúde (BR). Envelhecimento e saúde da pessoa idosa. Série A. Normas e manuais técnicos. Cadernos de Atenção Básica, n.19. Brasília (DF): 2007. 
21. World Health Organization. WHO global report on falls prevention in older age. 2007.

22. Li W, Keegan T, Sternfeld B, Sidney S, Quesenberry C, Kelsey J. Outdoor falls among middle-aged and older adults: a neglected public health problem. Am J Public Health. 2006 Jul; 96(7):1192-200.
23. Ribeiro A, Souza E, Atie S, Souza A, Schilithz A. A influência das quedas na qualidade de vida de idosos. Ciên Saúde Coletiva. 2008 Jul; 13(4):1265-73.

24. Pereira C, Vogelaere P, Baptista F. Role of physical activity in the prevention of falls and their consequences in the elderly. Eur Rev Aging Phys Act. 2008 Abr; 5(1):51-8. 